\title{
Conception and Development of a Dynamic Web Site: Dedicated to a University Library
}

\author{
Safia Nait Bahloul \\ Department of Computer Science, University of Es-sénia Oran-Algeria
}

\begin{abstract}
The world wide web is a group of web pages related between them with hypertext links. That forms a huge and complex web mail. A single click allow us to surf from document to another. From each document we can refer to different types of information: text, sound, video. In most cases, first sites were static, their principal aim were suggesting products and services. In the opposite, the web pages feed actually more opportunities to make dynamic contacts with users, with allowing data exchanges : from and to both sides (server and client).To establish this practices, distinct environment and tools were developed such as: Script languages, Database servers, ...etc. A Part of this paper study a practical case, which is: Dynamic conception of university library site, with explaining different tools and environment incorporate
\end{abstract}

Key words: Database, Internet, Site, World Wide Web, PHP, MySQL, Dynamic Web, Form

\section{INTRODUCTION}

The systems of classic information [1] limited themselves to local necessities of companies: effective access to data, competes of access, security and distribution on a network.

At present new necessities appeared. Companies or any automated organization wish to exchange its information and its services with the external environment (an unity untied from the same company, another company, a customer,) what gave birth to the notion of systems of information opened [1]. The evolution of the data processing in material software and material and the appearance of new technologies such as Internet [2] answer pertinently these necessities. What stood out with more this last decade in the field of the systems of information, it is well this new tool of communication: Internet. This last one allows the users to send electronic messages worldwide complete, to exchange economic data, multimedia data (sounds, images,) and to surf on World Wide Web [2].

World Wide Web is a set of web sites connected among them by hypertext links forming an enormous cobweb. A simple click of mouse allows us to surf of a document Web in the other one, while calling texts, images, videos, sounds, etc. [3].

In their big majority, the first sites were static. Their main end was to propose information about products and services. Contrary to this practice, the current web sites offer more and more the possibility of entering interaction with the users by allowing to exchange in two senses data among the customer and the server: dynamic Web [4]. To assure this practice, various environments are developed such as the languages of script and server of database [5].
A part of this article consists in a study of actual case, on which we put into practice the various notions of Web, which is the conception of a dynamic Web site for the library of the university of Oran's Es-Sénia by defining various working environments.

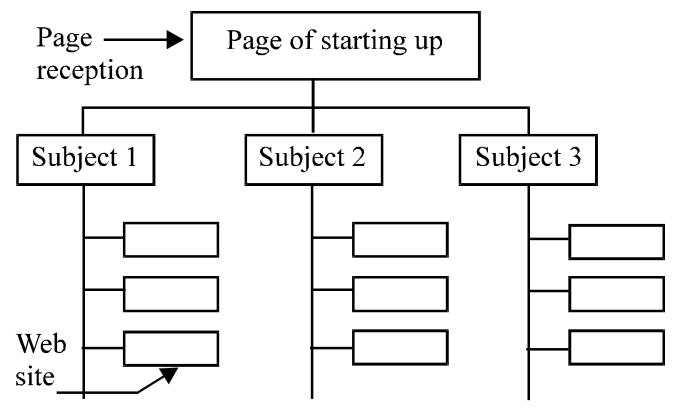

Fig. 1: Structure of a Site

The Architecture of a Web Site: A Web site consists of one or several web sites being able to contain of the text, the images and the other interconnected elements. The front page of a site is called page of starting up or home page [3] (Fig. 1).

Web sites are coded by the language Hypertext Markup language (HTML) [6, 7]. It is about a language of pagination of documents and specifications of links which the navigator will analyze to show the contents of the document, including the text, the images and the other types of entities. This language offers also a means to realize interactive documents by using hypertext links, what allows to connect our document with the other documents, that they are on our system or somewhere on Web [5].

The Language of Universal Script for Web

Definition: Hypertext Preprocessor (PHP) [8, 9] is a language of quoted script server, he is used with 


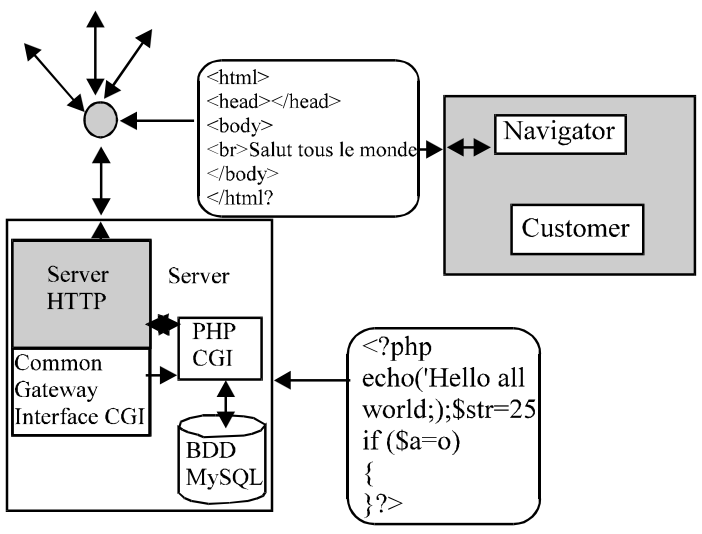

Fig. 2: $\quad$ PHP as an CGI

Internet servers to create with a minimum of effort of the dynamic web sites intended for Internet applications multimedia or of e-business. The code of script is executed by the server in answer to a request Hypertext Transfer Protocol (HTTP) [8, 10].

PHP's syntax is inspired widely by the language $C$, while presenting certain lines of relationship with languages Perl [11] and Java. [12] PHP is a language multiplate-forms: it was concerned numerous stations UNIX, such as Linux, and it so well on machines Windows [8].

PHP can be used or as a program portable CGI is as a module integrated into the server Web as Apache [13] (Fig. 2). What returns interesting language PHP is the faculty in interfacer in of numerous data base SQL [14] and the direct coverage of several server of management of database (DBMS) as:Adabase D, Dbase, Msql, Oracle... [8]

PHP's Functioning: Code PHP can be an integral part of the HTML page or a pure script PHP. The user who calls such a page ignores everything on the code of this page, this code having been interpreted quoted server before being translated into code HTML. To do it, the server web throws the interpreter PHP, who translates the wanted document and executes code source of the page. Command appearing in the page are interpreted and result takes the shape of a HTML page published in the place of the code source in the same document.

At the conclusion of this phase of translation, the modified page is sent to the customer to be shown there by the Navigator [8].

Treatment of Forms in PHP: The point of departure of the programming of interactive web sites is constituted with forms HTML, these last ones constitute an interesting solution to transfer data of the customer towards the server http. One of the reasons of the success of the language PHP is that scripts allow to manipulate very easily the data of forms. It is dregs in the fact that every name indicated in a form to identify a zone of seizure HTML is automatically transformed into a variable by PHP. The general structure of a script of treatment of form owes (Fig. 3a and b):

* To prepare the very form;

* To proceed to controls of seizure (PHP uses for it regular expressions);

* To make actions as seizure is confirmed or not;

MySQL's General View: MySQL $[8,15]$ is a language of requests used for the interrogation of the data base relational [14], MySQL uses architecture multi-user, multiprocessing.

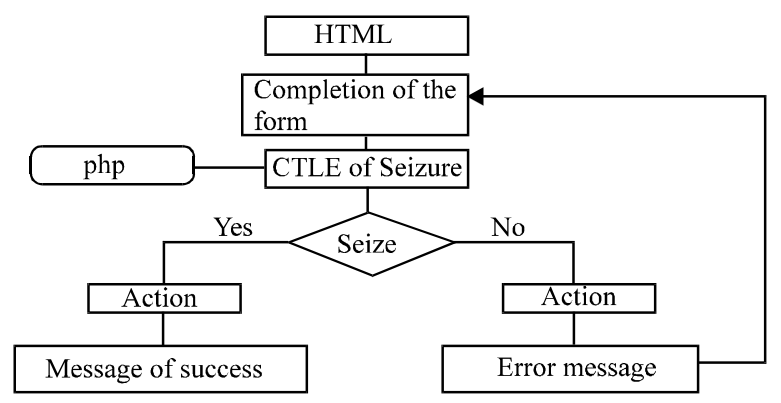

Fig. 3a: Treatment of Form in PHP

I*The skeleton of a script of treatment of form uses:

CTLE: control: The control of seizure makes by a part of the script, if seizure is valid one will continue the execution of the script otherwise an error message and the form seized first will have returned.*/

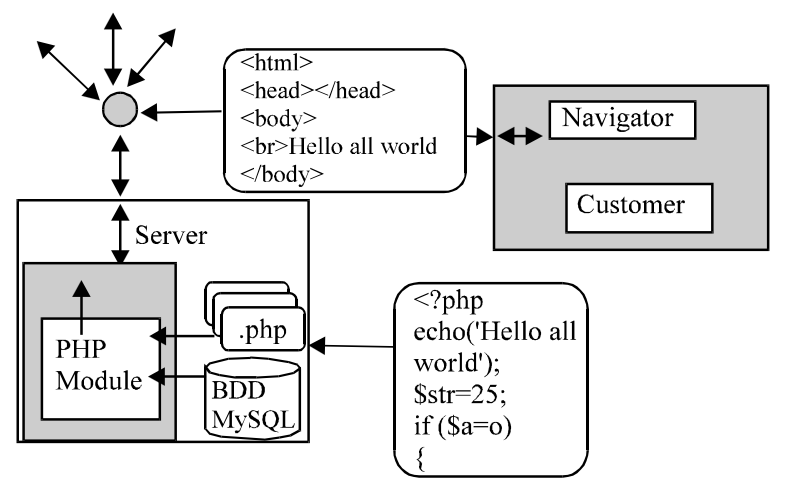

Fig. 3b: PHP as an Apache Module

It allows to establish fast connections and to use the same memory hide for several requests [8].

MySQL's main features:

* Architecture multiuser and multiprocessing

* Taken care of several flat forms of operating system

* Function(office) SQL strongly optimized

* The tables of various data bases can be used in a request 
* Taken care with data base of very big size

* Connection among the waiter(server) of data base MySQL and the customers passes by connections TCP / IP.

Administration of the Server of Data Bases MySQL from the Navigator Web: The use of the customer mysql does not seem pleasant. The customer lacks conviviality, the possibilities of publishing in case of error of seizure are rather unpolished. The use of line of command does not arrange the users familiarized with graphic interfaces. There are however various utilities making friendlier the administration of data base MySQL. One of these utilities, phpmyadmin, consists of a set of scripts PHP, allowing to administer data bases MySQL by way of a navigator Web. This utility makes indeed a particularly striking demonstration of the access to data bases MySQL by means of PHP [8].

Programming Data Bases Under PHP and MySQL: Typical scenario for the use of a data base MySQL with PHP appears as follows :

The server of data base MySQL is situated on the server distant from the access provider with Internet (FAI). This one attributes an user account, a password and the straight correspondents which allows to administer the server of database.

Access to the server of data base MySQL by using scripts PHP, allows to create the databases, tables, to insert data into tables and to interrogate bases by means of suited requests. For this to make PHP have a whole series of functions intended for the access to a server of data base and the manipulation of data [8]. MySQL's use with PHP is made in four stages:

* Connection to the server of data

* Selection of the base of data

* Request

* Exploitation of requests

Case Study: This case study consists in a conception and a realization of a dynamic Web site dedicated to the library of Oran's university one using MYSQL and PHP. The main tables which contains a library (considered as a database) are: Reader, Book and Loan OnLine [16].

* The table Book contains following attributes: Code, NameAut (name of the author), Titles, Edition, NbrPge (number of pages), NbrExp (number of copies), Disp ( availability).

* The table Reader contains following attributes: NumRead, Name, First name, Street, City, Codepostal, Country, Telephone, Email.

* The table Loan OnLine contains following attributes: NumLoan, NumRead, Code, Date, Restores

Tables required for the data base can be create directly to the guest of the customer mysql mysql. It turns out however more simple to write in a file ASCII SQL commands required in this end and to send this file to the server mysql by means of the utility of administration PhpMyAdmin [8].

Completion of Tables: After the creation of the data base and the tables, their completion makes through the utility PhpMyAdmin.

Example: Completion of the table Book

INSERT INTO Book (Code, NameAut, Title, Edition, NbrPge, NbrExp, Disp) VALUES ("I774", "COCHET Claude", "Analyse Organique en Informatique de Gestion ", "Paris : Dunod Informatique", 156, 2, 2 ); // Insert into the table Book values for every attribute

General Architecture of the Site: In the entrance of the site we shall have a page of reception which contains a static part (a set of web sites connected among them by hypertext links) and three dynamic columns: Registration, Loan, Search by keyword (Fig. 4).

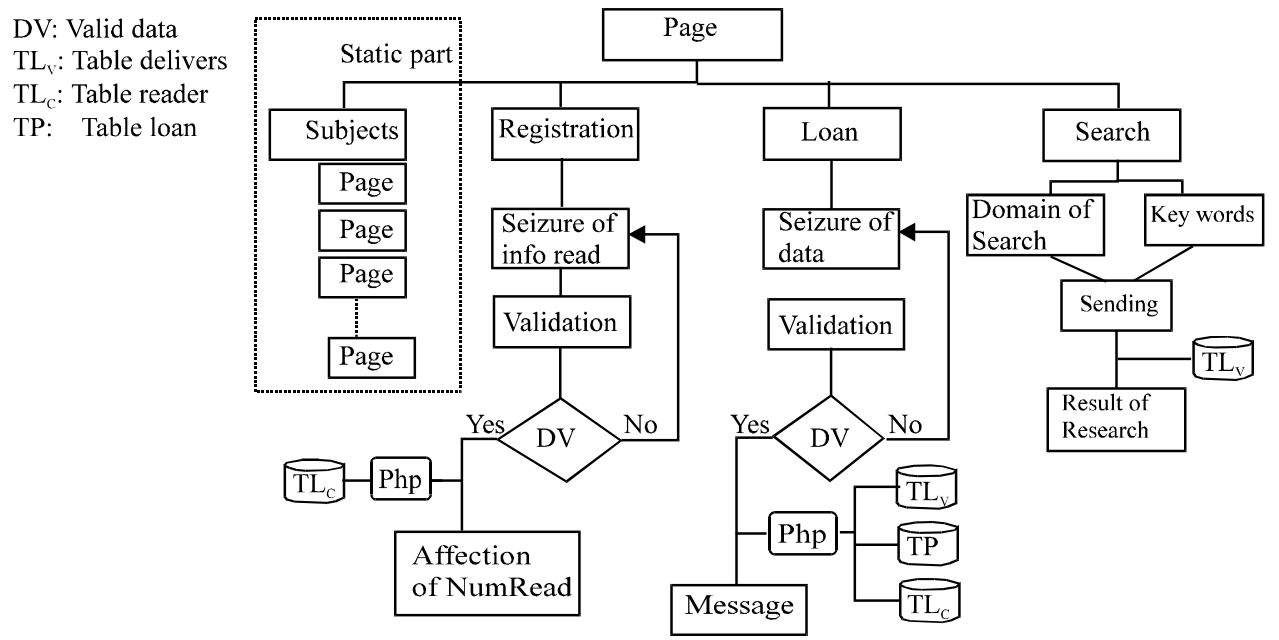

Fig. 4: General Architecture of the Site 
Navigation on the Site: A simple click on one of subjects (Data processing, Chemistry, Biology, Mathematical, physical) will lead us to another page which contains the in order alphabetical organized set of the works of titles.

A click on a letter leads us or at the beginning of the works which begins with this letter in the same page (local link) or on the another page situated in the another file Html ( external link) (Fig. 5).

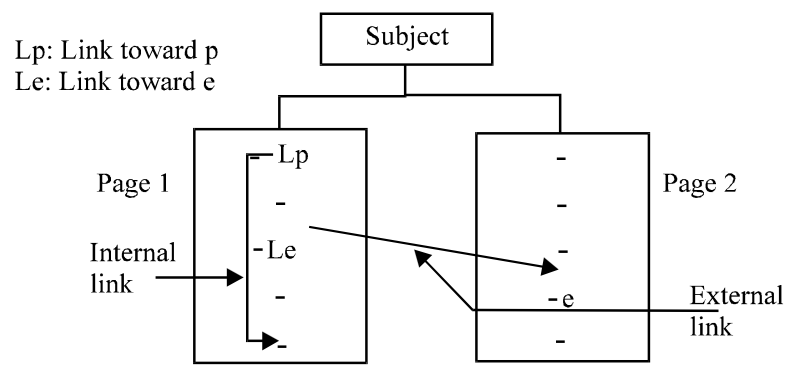

Fig. 5: Hypertext Link

Dynamic Part of the Site: The columns of this part allow to exchange data with the server Web Apache, this last one is in direct relation with the server of data base MySQL. Connection among the customer and the server is made through the language of script PHP which gets back the data of the user through a form, emits requests and get back results which will be posted on the navigator.

Our application contains three important columns of this genre: search by keyword, registration and loan Online whom we are going to detail in what follows.

To interrogate a data base it is necessary at first to connect to the server MySQL, it makes by the following function: int mysql_connect

(string hostname: port: connation, string username, string password)

\section{Example:}

$<$ ?

if( mysql_connect(localhost, ' ' ,' ' ) > 0 )//localhost :

The machine used

as server and customer at the same time

echo ' Successful connection!' ;

else

echo ' Impossible Connection !' ;?>

In what follows we are going to explain the procedures of the dynamic columns which are Registration in the library, loan Online and Search by keyword

Registration: Before making a loan, the reader at first has to join the library by filling the form of registration. Various cases are envisaged (Fig. 6).

After the completion of a form we proceed to the control of seizure, PHP uses for it functions which use regular expressions. Let us take the example of the validation of an email:

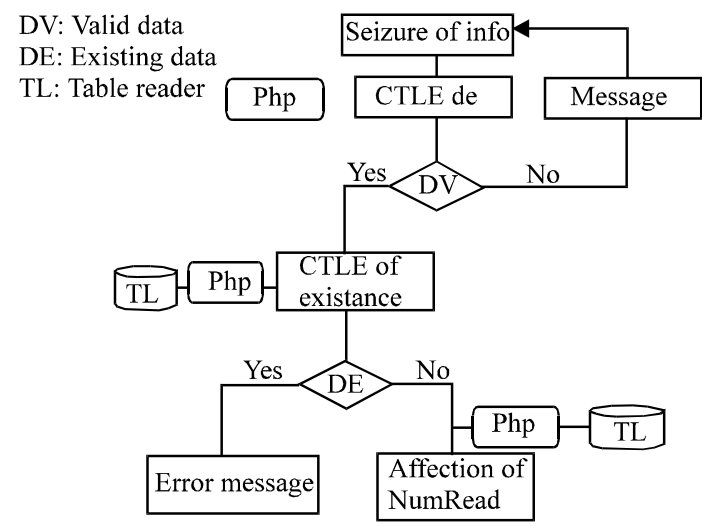

Fig. 6: Registration in Library

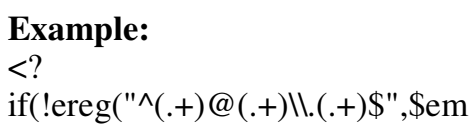

ail))

echo ('Valid email');

else

echo ('Not valid email ');

?>

Here is a portion of the script of registration in the library]

$<$ ? .........

// Treatment made in the case of success (the data of the form are valid)

// The case where the reader already exists

\$sql3="SELECT * FROM Reader where Name $=$ '\$

Name' and First name ='\$ First name ' ";

\$res3=send_sql( $\$ \mathrm{db}, \$$ sql3);

\$nombre1=mysq1_num_rows (\$res3);// The number

of readers in the table

if $(\$$ nombre $1 !=0)\{/ /$ The reader is already registered.

An error message will have returned\} else $\{/ /$ The case of a new registered // Update of the BD

\$sql="INSERT INTO 'lecteur' ('NumLect','Name','First name', Street,'City', 'codePostal','Telephone ',Email')

VALUES ('\$NumLect', '\$name', '\$first name','\$street','\$city',

'\$telephone','\$email')";

\$result_sql=send_sql(\$db,\$sql);

// Affectation of $\mathrm{n}^{\circ}$ of registration

\$sq12="SELECT NumRead FROM Reader where Name='\$name' and Email='\$email"';

*

\$result_sql2=send_sql $(\$ \mathrm{db}, \$ \mathrm{sql} 2)$;

else $\{$ // Data are not valid

An error message will have returned by indicating various place erroneous

\}?>

Loan Online: Loan makes by filling the form of the loan and after the sending of this form, various cases are envisaged (Fig. 7): 


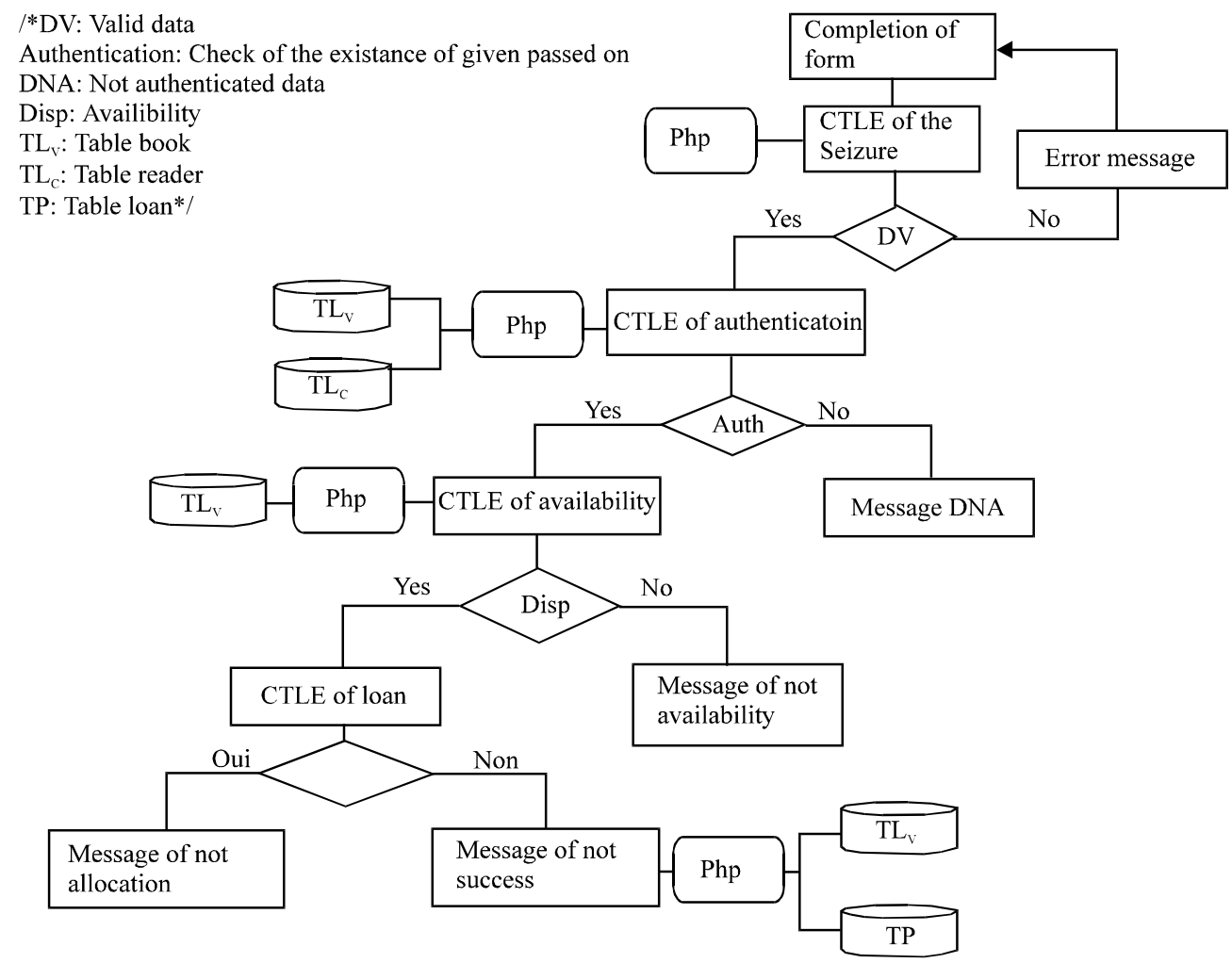

Fig. 7: Diagram of Loan Online

The script of loan:

$<$ ? .........

// Update of the BD

\$sql1="select $*$ from Reader where

NumRead='\$numread"';

\$sq12="select * from Book where Code='\$code'";

$\$$ sql3="select $*$ from loan OnLine where

NumRead='\$numread' and

Restore ='nane' ";

\$res1=send_sql(\$db,\$sq11);//send_sql Send and verify request

\$res2=send_sql $(\$ \mathrm{db}, \$ \mathrm{sq} 12)$;

\$res3=send_sql $(\$ d b, \$ s q 13)$;

\$nombre1=mysql_num_rows (\$res1);// return the number of recordings

\$nombre2=mysql_num_rows (\$res2);

\$nombre3=mysql_num_rows (\$res3);

if ( nombre1==0) \{// Reader is not registered

echo" You are not Registered. You have at first to pass

by the stage of registration before making loan ";

elseif ( $\$$ nombre2==0) $\{/ /$ The book does not exist

echo" Sorry the library does not

contain the sought book ";

\}

elseif $(\$$ nombre3 $>=2)\{/ /$ The reader made more than two loans

echo" You can not make the loan of more than two books";

\}

else $\{/ /$ Control of availability \$row=0;

\$succ=mysql_data_seek $($ res2, $\$$ row $)$;
\$arr=mysq1_fetch_array(\$res2,MYSQL_ASSOC);// return result to a array

\$var $=\$ \operatorname{arr}[$ Disp];

if $(\$ \operatorname{var}==0)\{$

echo" Sorry The book which you sought is not available for the moment please renew your demand soon ";

$$
\text { \} }
$$

else $\{/ /$ Available book

\$var=\$var-1;

\$maj1="UPDATE Book set Disp='\$var'

where Code='\$code'";// Update of

the table Deliver

\$result=send_sql( $\$ \mathrm{db}, \$$ maj 1$)$;

$\$$ maj2="insert into loan OnLine

values('\$NumPret', '\$numread','\$code',

curdate(), 'non')";

\$result2=send_sql( $\$ \mathrm{db}, \$$ maj2);

echo" Success. You go to receive your book Very

soon ";

?>

Search by Key word : In the page of reception a form of search is inserted. Search makes as follows :

* Selection of the domain of search (by Author, Title or Edition)

* Introduction of the keyword of search.

* After the validation a result of search will have returned under shape of a array which contains the 
works which answer data introduced into the form of search.

Script of the search by keyword:

$<$ ? .

if (\$type==' Author ') \{

\$sql="SELECT DISTINCT * FROM Book

WHERE NomAut LIKE '\%\$SearchString\%' ORDER

BY code";

\$res=send_sql( $\$ \mathrm{db}, \$ \mathrm{sql})$;

\$nombre=mysql_num_rows (\$res);

// The case of the search did not return anything

if $($ nombre $==0)\{$

echo nombre;

echo" No result corresponds to your

keyword of search "; \}

// The case of the search returned of result else \{ echo ' The number of books which corresponds to your search is '; echo \$nombre; tab_out(\$res,\$Identifiant);//

return result under shape of a array ?>

\section{CONCLUSION}

Work made in this study joins within the framework of the conception and the realization of the systems of information, in particular Web applications. We gave general outline on Web, then we showed how creates web sites and how to make links among them, it by using the standard language of Web which is the HTML under the professional editor 'WebExpert' as well as the use of the language of script PHP and the language of requests MySQL to give a dynamic aspect to the site.

We showed also the power of these two tools and we explained the functioning of dynamic Web: of the launch of the request http of highly-rated customer in the execution of scripts, the interrogation of data until the recovery of the page result.

Finally we put into practice these various notions in a study of the case of a library of the university, by explaining the various stages of realization.

\section{REFERENCES}

1. Le Coadic, Y.F., 1998. Le Besoin d' Information: formulation, négociation, diagnostic. Paris, Edition ADBS.

2. Kretschmer et W. Gieseke, B., 1996. Le Grand Livre Internet. Edition. Micro Application.

3. Muller, P., 2000.Internet: Créer votre site Web. Edition. Micro Application.

4. Rochenmann, J.P., 2004. l'Audit du Site Internet. Edition Eyrolles.

5. Spainhour et V. Quercia, S.,1996. Web Master in a Nutshell. Edition. O'Reilly.

6. Raggett, D., A. Le Hors and I. Jacobs, 1998. "HTML 4.0 Specification" The 24 April 1998 version is http://www.w3.org/TR/1998/REChtml40-19980424. The 24 April version included editorial changes from the original 18 December 1997 Revision.

7. WAI. Web site: http://www.w3.org/WAI/Guidelines for designing accessible HTML documents are available at the Web Accessibility Initiative.

8. Leierer et R.Stoll, G.A., 2000. Le Grand Livre PHP4/MySQL. Edition. Micro Application.

9. http://www.PHP.net. Documentation, Téléchargement: PHP, MySQL.

10. Fielding, R., J. Gettys, J. Mogul, H. Frystyk Nielsen, L. Masinter, P. Leach and T. Berners-Lee, 1999. "Hypertext Transfer Protocol -- HTTP/1.1". This RFC obsoletes RFC 2068.

11. Wall, L., 2004. Progrmmation en Perl. $2^{\text {nd }}$ Ed., Edition O'Reilly.

12. Macary, J.F. and C. Nicolas, 1983. Progrmmation Java. Edition Eyrolles.

13. Laurie, B., 2003. Apache: The Definition Guide. $2^{\text {nd }}$ Ed., Edition O'Reilly.

14. Codd, E.F., 1970. A Relational Model of Data for Large Shared Bank. Cacm, 13: 377-387.

15. http://www.mysql.com. PHP.NET Le site officiel de PHP.

16. Ouamara, A., 2002. Création et hébergement d'un site Web dynamique dédié à la bibliothèque de l'université d'Oran.Université d'Es-Sénia d'Oran-mémoire d'ingénieur, IGMO-, pp: 99. 